\title{
Quantum-Well Wavefunction Localization and the Electron-Phonon Interaction in Thin Ag Nanofilms
}

\author{
S. Mathias, ${ }^{1}$ M. Wiesenmayer, ${ }^{1}$ M. Aeschlimann, ${ }^{1}$ M. Bauer ${ }^{2}$ \\ ${ }^{1}$ Department of Physics, University of Kaiserslautern, 67663 Kaiserslautern, Germany \\ ${ }^{2}$ Institut für Experimentelle und Angewandte Physik, Universität Kiel, 24098 Kiel, Germany
}

(Dated: August 8, 2018)

\begin{abstract}
The electron-phonon interaction in thin $\mathrm{Ag}$-nanofilms epitaxially grown on $\mathrm{Cu}(111)$ is investigated by temperature-dependent and angle-resolved photoemission from silver quantum-well states. Clear oscillations in the electron-phonon coupling parameter as a function of the silver film thickness are observed. Different from other thin film systems where quantum oscillations are related to the Fermilevel crossing of quantum-well states, we can identify a new mechanism behind these oscillations, based on the wavefunction localization of the quantum-well states in the film.

PACS numbers: 73.50.Gr, 73.21.-b, 71.18.+y, 79.60.Dp
\end{abstract}

Quantum oscillations of various physical properties in thin film quantum-well systems, which are controlled by the thickness of the overlayer film, have generated constant interest in recent years. A very prominent example are quantum oscillations in the superconducting transition temperature of thin $\mathrm{Pb}$-films with varying thicknesses [1, 2, 3, 4]. In the same context, quantum oscillations in the electron-phonon coupling have been observed in, for example, $\mathrm{Ag}$ and $\mathrm{Pb}$ [5, 6]. Additional examples include oscillations in the magnetic coupling [7], the stability of thin films [8], work-function [9] and electronic growth [10]. All these systems are characterized by the existence of discrete electronic quantum-well states (QW-states) and, up till now, any observed oscillatory behaviour has been related to a Fermi-level crossing of a QW-state at distinct film thicknesses. In this paper, we give first evidence for a different mechanism in thin film systems capable of promoting quantum oscillations. We show that quantum oscillations in the electron-phonon coupling of ultrathin Ag-films on $\mathrm{Cu}(111)$ are driven by transitions of a QW-state into a QW-resonance at distinct film thicknesses. This localization process enhances the probability of electron momentum transfer processes and, therefore, promotes electron-phonon scattering. In analogy to the relevance of Fermi-level crossing of QWstates we also expect this kind of wavefunction localization to alter a variety of relevant material properties in a similar quantum oscillatory way.

Our photoemission data were recorded at normal emission using a hemispherical energy analyzer for parallel energy and momentum detection $(\Delta \mathrm{E}<20 \mathrm{meV}, \Delta \phi<$ $\left.0.15^{\circ}\right)$. Frequency-quadrupled UV light $(\mathrm{h} \nu=6 \mathrm{eV})$ of the output of a small bandwidth $(h \nu=1.5 \mathrm{eV})$ pulsed Ti:Sapphire laser system was focused onto the surface to a spotsize of approximately $150 \mu \mathrm{m}$. Silver films were grown at room temperature followed by short flash of the sample to $600 \mathrm{~K}$ 11]. Figure 11 displays a $E\left(k_{\|}\right)$Photoemission map of a 36 ML thick silver film recorded at a sample temperature of $211 \mathrm{~K}$ (intensity in gray scale as a function of energy and parallel momentum).
The spectrum shows an intense signal from the Shockley surface-state just below the Fermi-level at a binding energy of about $40 \mathrm{meV}$. The spectral features at binding energies of $390 \mathrm{meV}, 600 \mathrm{meV}$ and $840 \mathrm{meV}$ are due to the $\nu=1, \nu=2$ and $\nu=3 \mathrm{QW}$-states localized within the silver film overlayer [12, 13]. The appearance of these states is possible due to the presence of the sp band-gap of the $\mathrm{Cu}(111)$ substrate, which extends from $-4.25 \mathrm{eV}$ to $0.85 \mathrm{eV}$ binding energy at $k_{\|}=0 \AA^{-1}$. Figure $1 \mathrm{~b}$ shows a set of three photoemission spectra at normal emission for $16 \mathrm{ML}, 21 \mathrm{ML}$ and $28 \mathrm{ML}$ of $\mathrm{Ag}$ on $\mathrm{Cu}(111)$. In agreement with previous reports [12, 13], we observe a shift of the QW-states to lower binding energies with increasing silver coverage asymptotically approaching the energy of the upper band edge of the silver sp band (300 $\mathrm{meV}$ ). Furthermore, additional spectral features significantly below the lower edge of the sp band-gap ( $850 \mathrm{meV}$ ) are visible (gray shaded area). These are due to photoemission from quantum-well resonances (QW-resonances) in the silver film. These types of states are supported by a finite electron reflectivity at the silver/copper interface, which is due to wavefunction mismatch between the two materials and, in the present system, is enhanced by a lattice mismatch of about $13 \%$ between silver and copper [14. In contrast to pure QW-states, these states are obviously in resonance with the copper sp-band and couple to real electron states within the copper substrate. The coupling gives rise to extended penetration (delocalization) of these states into the copper going along with a reduction of the wavefunction amplitude within the silver film [15, 16]. Figure 1; shows results from a quantitative analysis of photoemission maps recorded at varying film thicknesses. Monotonous transitions of the $\nu=2, \nu=3$ and $\nu=4 \mathrm{QW}$-resonances (open squares) into QW-states occur at film thicknesses of about $23 \mathrm{ML}, 35 \mathrm{ML}$ and 47 ML. Additional evidence for the change in the character of these states at these film thicknesses is a narrowing of their linewidths right at these critical thicknesses in agreement with reference [16].

A calculation of the binding energy of the $\nu=1$ to $\nu=4$ 

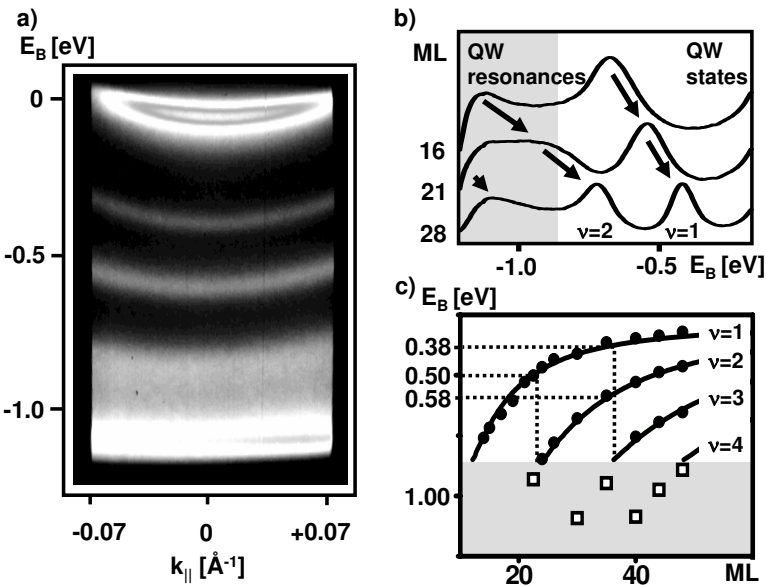

FIG. 1: (a) Typical 2D photoemission map for $36 \mathrm{ML}$ $\mathrm{Ag} / \mathrm{Cu}(111), T=211 \mathrm{~K}, h v=6 \mathrm{eV}$. (b) Transition of a QWresonance into a $\mathrm{QW}$-state with increasing Ag-thickness. The shaded area indicates the projected $\mathrm{Cu}$ bulk bands to the surface. (c) Energies of the $\nu=1$ to $\nu=4 \mathrm{QW}$-states (dots) and QW-resonances (squares) as a function of nominal film thickness [11]. The solid curves are calculations using the phase accumulation model with $m^{*} / m=0.65$, in agreement with data published before in 12]. The dotted lines link the QW-state QW-resonance transitions occurring at $23 \mathrm{ML}$ and $35 \mathrm{ML}$ to the binding energy scale.

QW-states was performed using the phase accumulation model following the details described in references 17] and [18] (see solid lines in figure 19). The model reproduces our experimental data perfectly for $m^{*} / m=0.65$ using an interfacial phase contribution given by [17, 19]

$$
\Phi_{C}(E)=2 \arcsin \sqrt{\left(E-E_{L}\right) /\left(E_{U}-E_{L}\right)},
$$

where $E_{L}=-0.85 \mathrm{eV}$ and $E_{U}=4.25 \mathrm{eV}$ are the lower and upper edge of the $\mathrm{Cu}(111)$ band gap 20], respectively. The linewidth $\Delta E$ of the $\mathrm{QW}$-state peaks is related to the lifetime of the photohole via the uncertainty principle and is governed to a considerable extent by electron-phonon interactions. An increased phonon population at elevated temperatures leads to an enhanced phonon scattering rate, and hence a shorter lifetime and an increase in the QW-state linewidth. Figure 2 2 shows photoemission spectra of the $\nu=1$ and $\nu=2 \mathrm{QW}$-states for a $36 \mathrm{ML}$ thick silver film at temperatures of $211 \mathrm{~K}$ and $336 \mathrm{~K}$ (open squares). The two Lorentzians result from fits of the QW-states while the solid lines represents the total fit of the spectra. Figure $2 \mathrm{~b}$ summarizes for this film the linewidths of the $\nu=1$ and $\nu=2 \mathrm{QW}$-state and the Shockley surface state as a function of temperature between 200 and $350 \mathrm{~K}$. An important parameter that can be directly deduced from these data is the electronphonon coupling parameter $\lambda$ [21], which is given by

$$
\lambda=\frac{1}{2 \pi k_{B}} \frac{d(\Delta E)}{d T} .
$$
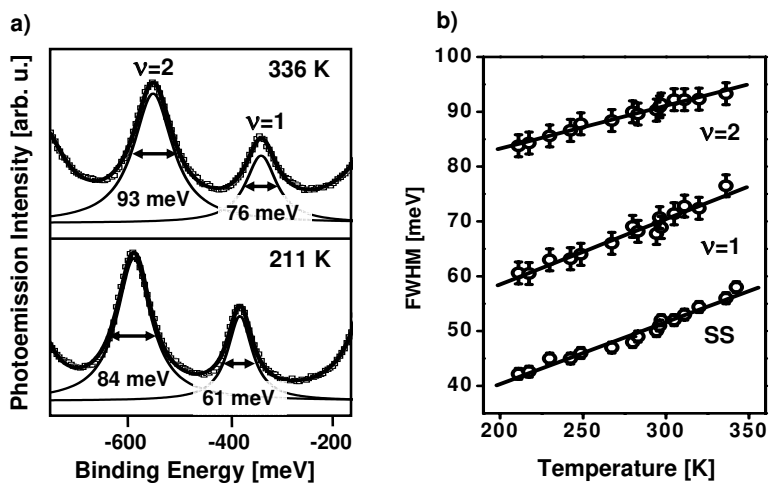

FIG. 2: a) Temperature dependent photoemission spectra of $36 \mathrm{ML} \mathrm{Ag} / \mathrm{Cu}(111)$ for $336 \mathrm{~K}$ and $211 \mathrm{~K}$. The $\nu=1$ and $\nu=2$ peaks shift in binding energy and the linewidth increases with increasing temperature. b) Linewidths (dots) and linear fit (solid line) of the QW-states and the Shockley surface state as a function of temperature for this film.

$d(\Delta E) / d T$ is the slope of the observed linear temperature dependence. The electron-phonon coupling parameter clearly depends on the quantum number $\nu$ of the QW-states.

Next, Figure 3 shows electron-phonon coupling data obtained from QW-state linewidth measurements for silver films of $15 \mathrm{ML}$ to $36 \mathrm{ML}$ thickness. The data are displayed here as a function of the QW-state binding energy. Full and open circles correspond to the values obtained for the $\nu=1$ and $\nu=2 \mathrm{QW}$-states, respectively. The general trend observed is an overall decrease of $\lambda$ with decreasing binding energy (solid lines). This trend is, however, interrupted by steps which are most pronounced at QW-state binding energies of about $0.50 \mathrm{eV}$ and $0.38 \mathrm{eV}$ for the $\nu=1$ state, but are also visible at a binding energy of $0.58 \mathrm{eV}$ for the $\nu=2$ state.

The linear decrease is consistent with a simple model previously used to quantitatively describe results for the $\mathrm{Ag} / \mathrm{Fe}(100)$ system [5]. This model allows us to assign the binding-energy dependence of $\lambda$ to the phase shift variation of $\pi$ across the confinement gap at the silver/copper interface [17] (see equation (11)). This phase shift $\Phi_{C}(E)$ modulates the amplitude of the QWstate's wavefunction at the interface and, therefore, affects the coupling efficiency of the QW-states to interfacial phonons. Since this modulation is only dependent on the binding energy of the QW-states, the behavior within our film thicknesses is independent of the quantum number $\nu$.

However, the experimentally observed deviations from this linear trend at $0.50 \mathrm{eV}$ and $0.38 \mathrm{eV}$ binding energy $(\nu=1)$ and at $0.58 \mathrm{eV}$ binding energy $(\nu=2)$ cannot be reproduced by this model. The observed step-like behavior requires a corresponding change in the interfacial phase contribution, which is not existent for the investigated 


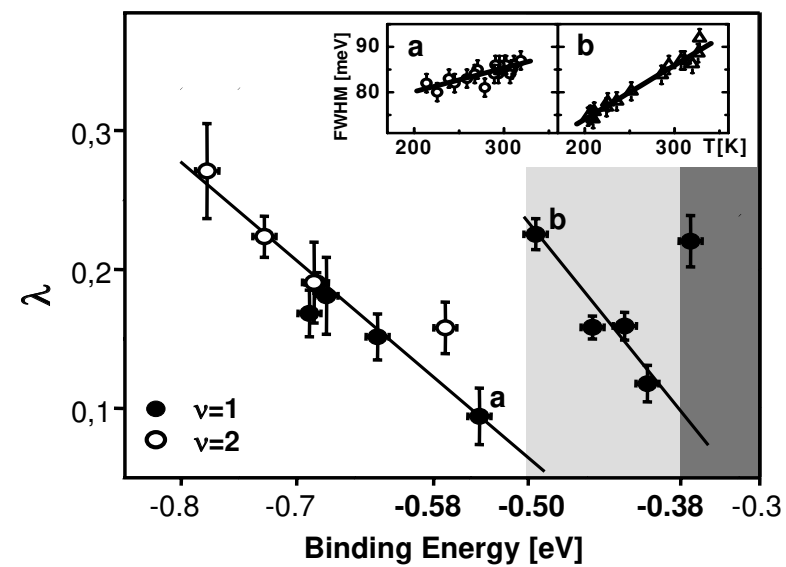

FIG. 3: Electron-phonon coupling parameters for the QWstates $\nu=1$ (full circles) and $\nu=2$ (empty circles) as a function of binding energy, deduced from T-dependent linewidth measurements as shown in the inset and Figure 2 $\mathrm{b}$. Oscillations in $\lambda$ occur at binding energies (bold printed) at which a QWresonance to QW-state transition occurs, as can be seen in Figure 1c. For reference, the gray shaded areas mark the binding energy regimes of increased electron-phonon scattering due the $\nu=1$ to $\nu=2 \mathrm{QW}$-state interaction (light gray) and due to the $\nu=1$ to $\nu=3 \mathrm{QW}$-state interaction (dark gray). The inset shows the temperature dependence of the linewidth of the $\nu=1 \mathrm{QW}$-state right before (a) and after (b) the $\lambda$-step at $0.5 \mathrm{eV}$ binding energy.

$\mathrm{Ag} / \mathrm{Cu}$ system.

Oscillations in the electron-phonon coupling parameter as a function of quantum-well thickness have been observed for other quantum-well systems before [1, 2, 3, 3, 6, 7, 8, 9, 10]. In all these cases, the origin of the oscillations was related to a Fermi-level crossing of a QW-state. The driving force for this effect is the strong modulation of the occupied density of states near $E_{F}$ as the QWstates cross the Fermi-level with increasing film thickness. However, for the $\mathrm{Ag} / \mathrm{Cu}(111)$ system, this scenario can be excluded. At $k_{\|}=0 \AA^{-1}$ the QW-states asymptotically converge at the lower edge of the silver sp bandgap at $300 \mathrm{meV}$ binding energy, which is well below the Fermi-edge (see Figure 11). Therefore, a Fermi-level crossing is not possible at all film-thicknesses and consequently cannot cause the observed oscillations in $\lambda$.

We found instead a direct correlation between the appearance of these oscillations and the critical film thicknesses where the transitions from the $\nu=2$ and $\nu=3 \mathrm{QW}$ resonances into the associated QW-states occur. For instance, the discontinuity in the $\nu=1 \mathrm{QW}$-state at $0.5 \mathrm{eV}$ binding energy is seen at a silver film thickness of 23 ML, exactly where we observe the transition of the $\nu=2$ QW-resonance into the $\nu=2 \mathrm{QW}$-state (see dotted lines in Figure 1r). In addition, both the second step of the $\nu=1 \mathrm{QW}$-state at $0.38 \mathrm{eV}$ binding energy and the step seen in the $\nu=2 \mathrm{QW}$-state at $0.58 \mathrm{eV}$ binding energy correlate with the $\nu=3 \mathrm{QW}$-resonance transition into the $\nu=3 \mathrm{QW}$-state at $35 \mathrm{ML}$. We therefore conclude that the origin of the oscillations in the electron-phonon coupling parameter $\lambda$ for the $\mathrm{Ag} / \mathrm{Cu}(111)$ system are directly related to these changes in the electronic structure, which occur at specific film-thicknesses as a QW-resonance undergoes the transition into a $\mathrm{QW}$-state.

The electron phonon-interaction in the silver film is governed by the available decay channels, the available phase space for each decay channel, and the matrix elements for the scattering processes [21, 22, 23]. The electron-phonon scattering process responsible for the phonon-mediated hole relaxation probed in the photoemission experiment requires, in particular, a source of electron momentum to compensate for the momentum of the absorbed or created phonon. Highly efficient in this means are QW-states interband scattering processes between two QW-states of different quantum number $\nu$ (see Figure $4 \mathrm{~b}$ ). This has been previously shown, for example, in the case of $\mathrm{d}$ band QW-states relaxation [23] and, in a similar manner, in connection with the decay of image potential states at $\mathrm{Cu}(100)$ surfaces [24]. An important contribution to the matrix element of such a process is the wavefunction overlap between the two involved QW-states.

In this context, we now consider in more detail the potential decay channels available for the $\nu=1$ hole created by the photoemission process (see Figure 4). Three different phonon-mediated scattering processes can be discriminated: the interaction of the $\nu=1 \mathrm{QW}$-state hole with (i) the $\mathrm{Cu}$ bulk-bands and, depending on the silver film thickness, the interaction with (ii) the QWresonance (S/R process, see Figure $4 \mathrm{H})$ ) and the interaction with (iii) the $\nu=2 \mathrm{QW}$-state (S/S process, see Figure 45). Due to the negligible wavefunction overlap between the $\nu=1 \mathrm{QW}$-state confined in the silver film and the bulk states of the copper substrate, the contribution of process (i) to the electron-phonon cross-section is of negligible importance. However, for processes (ii) and (iii), both the initial hole state ( $\nu=1 \mathrm{QW}$-state) and final hole state $(\nu=2 \mathrm{QW}$-resonance and QW-state, respectively) exhibit a considerable wavefunction amplitude within the silver film that promotes the electronphonon interaction. However, the leakage of the QWresonance into resonant $\mathrm{Cu}$-bulk states notably reduces its wavefunction amplitude in the silver film in comparison to the $\nu=2 \mathrm{QW}$-state. Indicative of this leakage are the low intensity and the large linewidth $\Delta E$ of the $\nu=2$ QW-resonance above a value of $400 \mathrm{meV}$ at $300 \mathrm{~K}$ (Figure 1 $\mathrm{b}$ ). From this point of view, the phonon-mediated $\mathrm{S} / \mathrm{R}$ scattering process must be less efficient than the $\mathrm{S} / \mathrm{S}$ process and this enables us to understand the microscopic mechanism behind the step-like increase in $\lambda$ at the critical film thickness of 23 ML. For a silver film just below this thickness, the electron-phonon coupling is governed by a phonon-mediated $\mathrm{S} / \mathrm{R}$ scattering process. As the film thickness increases, the $\nu=2 \mathrm{QW}$-resonance is transformed into the $\nu=2 \mathrm{QW}$-state corresponding to 
a wavefunction localization and an increase in the wave function amplitude. Right above the critical film thick ness, the electron-phonon coupling is therefore mediate by the $\mathrm{S} / \mathrm{S}$ scattering process, which is characterized b an enhanced interaction probability as observed in th experiment. The oscillatory behavior in $\lambda$ is therefor expected at a periodicity given by the rate at which QW-resonance into QW-state transition takes place, i: perfect agreement with our observations at $23 \mathrm{ML}$ an $35 \mathrm{ML}$ film thickness. This argument is also valid fo the discontinuity of the $\nu=2 \mathrm{QW}$-state at $580 \mathrm{meV}$ bind ing energy corresponding to the $\nu=3 \mathrm{QW}$-resonance QW state transition at $35 \mathrm{ML}$ thickness.

The ratio of the efficiency between the $\mathrm{S} / \mathrm{R}$ scatterin process and the $\mathrm{S} / \mathrm{S}$ process can be quantified by an es timate of the wavefunction amplitude of the $\nu=2 \mathrm{QW}$ resonance relative to the $\nu=2 \mathrm{QW}$-state in the silve film. For a finite reflection coefficient $R_{\mathrm{QWR}}\left(R_{\mathrm{QWR}}<\right.$ 1) of the QW-resonance at the silver/copper interfact the ratio of the wavefunction amplitude between th QW-resonance and QW-state $\left(R_{\mathrm{QWS}} \approx 1\right)$ is given $\mathrm{b}$ $R_{\mathrm{QWR}} / R_{\mathrm{QWS}} \approx R_{\mathrm{QWR}}$. $R_{\mathrm{QWR}}$ can be deduced fror the measured linewidth $\Delta E$ of the QW-resonance. According to reference [25], $\Delta E$ depends on the number of monolayers $\mathrm{N}$ of the silver film, $R_{\mathrm{QWR}}$ and the mean free path $\mathrm{L}$ of the electrons,

$$
\Delta E=\Gamma \eta \frac{1-R_{\mathrm{QWR}} \exp (-1 / \eta)}{R_{\mathrm{QWR}}^{1 / 2} \exp (-1 /(2 \eta))}
$$

with $\eta=L /(N t), \mathrm{t}$ is the monolayer thickness and $\Gamma$ is the intrinsic linewidth of the QW-resonance. For $L \approx N t / 2$ [26] and $\Gamma=90 \mathrm{meV}$ as deduced from an extrapolation of the intrinsic linewidth data of the QW-state into the QW-resonance regime we obtain a value of the reflectivity $R_{\mathrm{QWR}}<1 / 4$. This means that the wavefunction amplitude of the QW-resonance is reduced in comparison to the wavefunction amplitude of the QW-state by a factor of about 4 . Also of the same order of magnitude is the ratio of the wavefunction overlap between the S/R and S/S scattering processes and, consequently, the expected ratio in the electron-phonon coupling constant. In this experiment, we find the ratio for the $\nu=1$ QW-state is equal to a value of about 3.5(1). Taking into account that the above estimation exclusively focuses on the effect of the wavefunction amplitude, the experimental and calculated values are in reasonable agreement. In conclusion, we have observed oscillations in the electron-phonon coupling parameter in thin Ag overlayer films on $\mathrm{Cu}(111)$. We have shown that these oscillations correlate with the transition of a QW-resonance into a QW-state in the silver film. Furthermore, we attribute the microscopic mechanism for these changes to the localization of the QW-state wavefunction within the silver film, which is going along with such a transition. In addition to quantum-oscillations in the electron-phonon

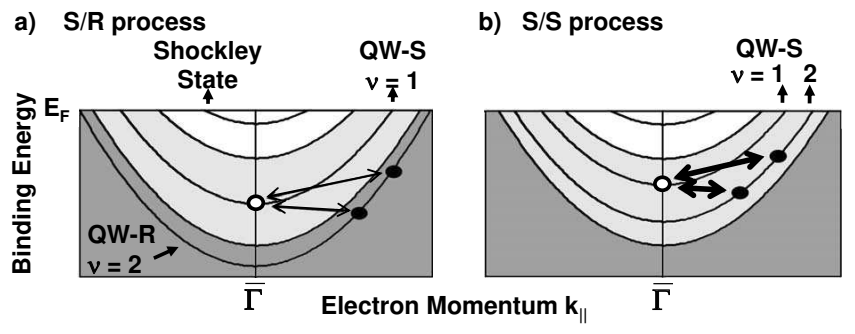

FIG. 4: Schematic illustration of the QW-state hole relaxation paths. Hole states are indicated by open circles, while electrons are indicated by solid circles. The dark shaded area is the projected bulk bands of the $\mathrm{Cu}(111)$-substrate to the surface. a) Phonon mediated scattering into the delocalized $\nu=2$ QW-resonance; b) enhanced electron-phonon coupling due to phonon-mediated interband scattering into the localized $\nu=2$ QW-state. The energy differences are highly exaggerated.

coupling parameter, we propose that this mechanism is of more general relevance for other thin film properties similar to the effect of the QW-state Fermi-level crossing. For example, one property for that oscillatory behavior has been shown in systems with oscillating $\lambda$ is superconductivity [1, 2, 3, 4, 27.

Special thanks go to M. Wessendorf. This work was supported by the DFG through SPP 1093 and the Stiftung Innovation RLP.

[1] Y. Guo et al., Science 306, 1915 (2004).

[2] D. Eom et al., Phys. Rev. Lett. 96, 027005 (2006).

[3] M. M. Özer et al., Nature Phys. 1, 117 (2005).

[4] T.-C. Chiang, Science 306, 1900 (2004).

[5] D.-A. Luh et al., Phys. Rev. Lett. 88, 256802 (2002).

[6] T. Valla et al., J. Phys.: Cond. Matter 12, L477 (2000).

[7] S. S. P. Parkin et al., Phys. Rev. Lett. 64, 2304 (1990).

[8] D.-A. Luh et al., Science 292, 1131 (2001).

[9] J. J. Paggel et al., Phys. Rev. B 66, 233403 (2002).

[10] Zhenyu Zhang et al., Phys. Rev. Lett. 80, 5381 (1998).

[11] S. Mathias et al., Appl. Phys. A 82, 439 (2006).

[12] M. A. Mueller et al., Phys. Rev. B 40, 5845 (1989).

[13] K. Takahashi et al., Phys. Rev. B 60, 8748 (1998).

[14] I. Meunier et al., Phys. Rev. B 59, 10910 (1999).

[15] M. Milun et al., Rep. Prog. Phys. 65, 99 (2002).

[16] T.-C. Chiang, Surf. Sci. Rep. 39, 181 (2000).

[17] N.V. Smith et al., Phys. Rev. B 49, 332 (1994).

[18] J. J. Paggel et al., Phys. Rev. B 61, 1804 (2000).

[19] M. H. Upton et al., Phys. Rev. Lett. 93, 026802 (2004).

[20] R. Paniago et al., Surf. Sci. 336, 113 (1995).

[21] G. Grimvall, The Electron-Phonon-Interaction in Metals (North-Holland, Amsterdam, 1981).

[22] P.M. Echenique et al., Surf. Sci. Rep. 52, 219 (2004).

[23] J. J. Paggel et al., Phys. Rev. Lett. 92, 186803 (2004).

[24] W. Berthold et al., Phys. Rev. Lett. 88, 056805 (2002).

[25] J. J. Paggel et al., Science 283, 1709 (1999).

[26] Ashcroft/Mermin, Solid State Physics (1976), fermivelocity in $\mathrm{Ag}: 1.39 \cdot 10^{8} \mathrm{~cm} / \mathrm{s}$.

[27] Y.-F. Zhang et al., Phys. Rev. Lett. 95, 096802 (2005). 